\title{
COVID-19 in CVID: a Case Series of 17 Patients
}

\author{
Jacob R. Greenmyer ${ }^{1} \cdot$ Avni Y. Joshi ${ }^{2,3}$ \\ Received: 19 May 2021 / Accepted: 4 October 2021 / Published online: 20 October 2021 \\ (c) The Author(s), under exclusive licence to Springer Science+Business Media, LLC, part of Springer Nature 2021
}

\section{To the Editor:}

Common variable immunodeficiency (CVID), caused by failure of B-cell differentiation with subsequent defective immunoglobulin production, presents heterogeneously as recurrent bacterial infections with increased propensity for autoimmune and malignant diseases [1]. SARS-CoV-2 infection has resulted in catastrophic respiratory, thrombotic, and inflammatory complications in many non-CVID patients [2]. Characterizing the risk factors for severe disease progression and identifying patient populations at risk are of the upmost importance to families and policy makers. Despite CVID being a relatively common primary immunodeficiency that predisposes patients to respiratory disease, little is known about the outcomes of patients with CVID who are infected with SARS-CoV-2 and how CVID subjects respond to SARS-CoV-2 vaccinations.

Here, we report the outcomes of CVID patients infected with SARS-CoV-2 during the COVID-19 pandemic at our institution.

Institutional review board approval for this study was obtained. We searched our electronic medical records for patients with concomitant diagnoses of CVID and SARS$\mathrm{CoV}-2$ infection. Twenty-three patient cases were reviewed using chart review methodology; 6 cases were excluded due insufficient criteria to meet the CVID diagnosis [2], hypogammaglobulinemia being the result of chronic lymphocytic leukemia [1], and limited data [1]. We summarized the cases of 17 CVID patients who were diagnosed with SARS-CoV-2 infection during the COVID-19 pandemic March 1, 2020-May 20, 2021, in Table 1. Severity of

Avni Y. Joshi

joshi.avni@mayo.edu

1 Department of Pediatric and Adolescent Medicine, Mayo Clinic, Rochester, MN, USA

2 Division of Allergy and Immunology, Mayo Clinic Childrens Center, Rochester, MN, USA

3 Rochester, USA
SARS-CoV-2 infection was classified using criteria provided by the NIH (https://www.covid19treatmentguidelines.nih. gov/overview/clinical-spectrum/).

Median age at diagnosis was 41 years (IQR: 26-48 years). There was a slight predominance of males $(59 \%, n=10 / 17)$ in this cohort. Supplemental IgG therapy was commonly prescribed in this patient group $(88 \%, n=15 / 17)$, with majority $(59 \%, n=10 / 17)$ on IVIG. All patients were PCR tested for SARS-CoV-2 and were positive. While the majority received no specific therapies, five patients (29\%) were hospitalized-three $(18 \%, n=3 / 17)$ of whom received convalescent plasma. Three patients $(18 \%)$ received monoclonal antibodies in the outpatient setting. All patients in our cohort survived, which was a very reassuring finding. Genetic analysis was performed on five patients as shown in Table 1.

Post COVID-19 infection, 29\% $(n=5 / 17)$ of the cohort received vaccination and tolerated it without significant adverse events. Post-vaccination serology was assessed in 1 patient, who demonstrated seroconversion with antispike antibody. Post-infection seroconversion was assessed in 6 patients and 5 of these patients $(83 \%, n=5 / 6)$ showed evidence of seroconversion at a median interval of 46 days (IQR: 30-98 days) post-acute COVID -19 infection. There were 2 patients $(12 \%, n=2 / 17)$ who reported persistent symptoms that were consistent with post-acute COVID sequalae (PASC). One patient with symptoms consistent with PASC had serology performed and showed no evidence of seroconversion. This may open the question of whether the lack of seroconversion is a marker of maladaptive immune response in CVID patients, which may predispose to PASC [3].

Despite immune deficiency posing a higher risk for COVID-19 infection, patients with CVID may be at a lower risk for hyperimmune/cytokine storm, which could explain a lower case fatality rate in the CVID patient population compared to patients with other chronic conditions [4].

This is one of the largest case series from the USA that describes the experience of a tertiary care center in managing high-risk patients with CVID and COVID-19 infection. These cases constitute approximately $20 \%(n=17 / 85)$ of 


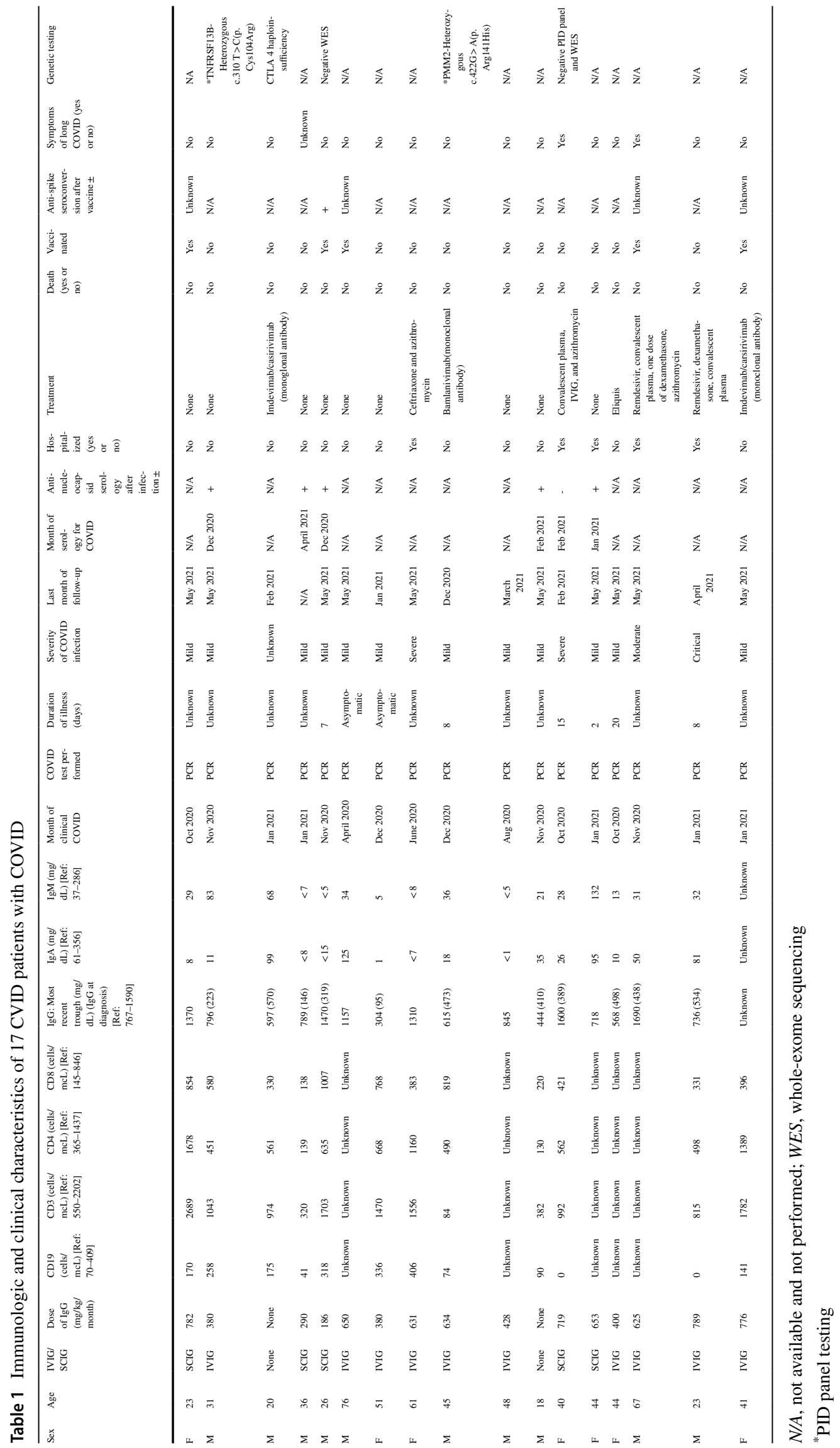


the reported cases of COVID-19 in CVID and help minimize reporting bias by reporting all available cases from our institution. Our institution has three unique strengths of management that may result in good outcomes: ready access to monoclonal antibodies, close follow-up of patients, and relatively good access to care.

This study is limited by being a small cohort but, of note, this is one of the earliest and largest case series of CVID in North America to date.

A recent review and quantitative analysis of coronavirus disease 2019 (COVID-19) infection in CVID patients found 14 publications on this topic and summarized 68 cases [5]. This important article by Weifenbach and colleagues reported that the number of patients with CVID with moderate to severe $(29 \%, n=20 / 68)$ infections was higher than the general public. Furthermore, they reported nine fatalities $(13 \%, n=9 / 68)$. The authors highlight that a major limitation of their review and the existing data is that asymptomatic and mild COVID-19 infections are mostly ignored; therefore, the study may overpredict true morbidity in CVID. Reporting bias may be reflected in the treatment these patients received: no treatment $(25 \%, n=17 / 68)$; antibiotics $(59 \%, n=40 / 68)$; chloroquine/hydroxychloroquine ( $47 \%$, $n=32 / 68)$; antiviral therapy $(21 \%, n=14 / 68)$; immunoglobulin $(10 \%, n=7 / 68)$; monoclonal antibody $(12 \%, n=8 / 68)$; and convalescent plasma $(12 \%, n=8 / 68)$. The authors concluded that larger-scale cohort reports including asymptomatic and patients with mild infection are needed to characterize the true risk of SARS-CoV-2 infection for CVID.

While our study is one of the largest North American studies of patients with CVID and SARS-CoV-2, its generalizability is limited by its observational design and small sample size. Regardless, there are two important observations and future directions from this data. First, in our institutional experience, COVID-19 disease course in CVID patients is similar to most other patients. The majority of our patients $(71 \%, n=12 / 17)$ had a mild disease course and all patients survived. The presence of CVID per se does not seem to increase the risk of mortality with COVID-19. This supports the idea from Weifenbach and colleagues that prior reports may be skewed towards disproportionately ill CVID patients [5]. Second, all 6 of our CVID patients who received the COVID-19 vaccine tolerated this without complications (to date), similar to our reported experience in overall immunodeficiency patients $[6,7]$. This study, while limited in its scope, is one of the first to comment on COVID-19 vaccine safety in patients with CVID. A related question about vaccine efficacy in patients with CVID is whether natural infection prior to vaccination boosts seroconversion rate with immunization. A registry or large-scale multi-institution study is needed to further validate these observations and questions. Given the relatively low incidence of CVID, a collaborative effort will be necessary to increase the strength of data and provide sound evidencebased recommendations to patients with CVID.

Author Contribution All the authors contributed to the study conception and design. Material preparation, data collection, and analysis were performed by Jacob R. Greenmyer and Avni Y Joshi. The first draft of the manuscript was written by Jacob R. Greenmyer and all the authors commented on previous versions of the manuscript. All the authors read and approved the final manuscript.

Data Availability Not applicable.

Code Availability Not applicable.

\section{Declarations}

Ethics Approval This study was approved by the Mayo Clinic Institutional Review Board and was performed in accordance with the ethical standards as laid down in the 1964 Declaration of Helsinki and its later amendments or comparable ethical standards.

Consent to Participate Not applicable.

Consent for Publication Not applicable.

Conflict of Interest The authors declare no competing interests.

\section{References}

1. Tam JS, Routes JM. Common variable immunodeficiency. Am J Rhinol Allergy. 2013;27(4):260-5.

2. Gupta A, Madhavan MV, Sehgal K, Nair N, Mahajan S, Sehrawat TS, et al. Extrapulmonary manifestations of COVID-19. Nat Med. 2020;26(7):1017-32.

3. Nalbandian A, Sehgal K, Gupta A, Madhavan MV, McGroder C, Stevens JS, et al. Post-acute COVID-19 syndrome. Nat Med. 2021;27(4):601-15.

4. Liu BM, Hill HR. Role of host immune and inflammatory responses in COVID-19 cases with underlying primary immunodeficiency: a review. J Interferon Cytokine Res. 2020;40(12):549-54.

5. Weifenbach N, Jung A, Lotters S. COVID-19 infection in CVID patients: what we know so far. Immun Inflamm Dis. 2021;9:6324. https://doi.org/10.1002/iid3.450.

6. Squire J, Joshi A. Seroconversion after coronavirus disease 2019 vaccination in patients with immune deficiency. Ann Allergy Asthma Immunol. 2021;127(3):383-4.https://doi.org/10.1016/j. anai.2021.05.015.

7. Squire JD, Joshi AY. Safety of COVID-19 Vaccination in immunedeficient patients receiving supplemental immunoglobulin therapies. J Clin Immunol. 2021;41(7):1527-30. https://doi.org/10. 1007/s10875-021-01101-8.

Publisher's Note Springer Nature remains neutral with regard to jurisdictional claims in published maps and institutional affiliations. 